Bull. Mater. Sci., Vol. 17, No. 6, November 1994, pp. 1103-1108. (C) Printed in India.

\title{
Some concepts on design of surfactant gels and vesicles
}

\author{
SUSHAMA MISHRA ${ }^{\ddagger}$, B K MISHRA ${ }^{\ddagger}$, D K CHOKAPPA ${ }^{\dagger}$, D O SHAH ${ }^{\ddagger}$ \\ and C MANOHAR \\ Chemistry Division. Bhabha Atomic Research Centre, Bombay 400 085, India \\ ${ }^{\dagger}$ llindustan Lever Research Centre. Chakala, Bombay 400 099. India \\ ${ }^{\ddagger}$ Department of Chemical Engineering. University of Florida, Gainesville, FL 32611, USA
}

\begin{abstract}
It is conjectured that anionic-cationic surfactant combination can be regarded as equivalent to a double chain surfactant and using molecular packing considerations it is shown that vesicles, viscoelastic solutions and liquid crystals can be designed by the proper choice of chajn lengths of the pair. Using these concepts new systens are designed, from nixtures of cetyltrimethyl ammonium bronude and sodium alkyl sulfonates, to produce both viscoelastic gels and vesicles.
\end{abstract}

Keywords. Vesicles; gels; surfactants; viscoelasticity.

\section{Introduction}

Dilute aqueous surfactant solutions are known to assume various structures like spherical micelles, cylindrical micelles, disc like micelles, tubules etc. and design of these are of interest to a number of applications in detergent, paint, drug delivery and other industries (Fendler 1982). Classic work of Tanford (1972), Israclachivili, et al (1976) and others (Nagarajan 1986) identified that the key parameter is the ratio of the volume of the hydrophobic chain $v$ to the product of length $l$ of the chain and the area $a$ of the polar head i.e., v/al. It has been shown that

$$
\begin{array}{ll}
v / a l=1 / 3 & \text { for spherical micelles, } \\
1 / 3<v / a l<1 / 2 & \text { for cylinders, } \\
v / a l>1 / 2 & \text { for disc or vesicles. }
\end{array}
$$

These considerations can act as convenient guidelines and surfactants can be designed to produce these structures. Some of the examples are using salt to convert spherical to cylindrical micelles (Mysels and Princen 1959) or use of double chain surfactants to produce vesicles (Bangham 1965) or nematic liquid crystals (Hertel and Hoffmann 1984). Recently Kaler et al (1989) introduced a novel concept of combining a cationic and an anionic surfactant to spontaneously produce vesicles. Their results also showed that the charge on the vesicles can be controlled by this method of preparation. This aspect could be of great interest in cases where one needs a specific binding to charged surfaces. Their phase diagram also showed that the coulomb interaction between the vesicles plays an important role.

The present paper originated from the observation that the charge effects play a decisive and dominating role in obtaining viscoelastic gels of cylindrical micelles (Mishra et at 1993) and that the ideas which led Kaler et at (1989) for vesicles can be extended to viscoelastic gels too. Viscoelastic surfactant solutions have been 
reviewed in an excellent article by Rehage and Hoffmann (1991) recently which gives an exhaustive review of the experiments and a summary of available understanding of the subject. Some of the main characteristics which are exhibited by a number of systems but not understood are: (a) The zero shear viscosity of CTAB or other cationics shows two peaks as the additive such as sodium salicyate is added, (b) the minimum in the viscosity between the two peaks occurs approximately at 1:1 ratio of the two components or at slightly higher values of the concentration of the aromatic additive, (c) very often phase separations occur in the region between the two peaks and in some cases liquid crystalline phases occur in addition to phase separations (Rehage and Hoffmann 1991; Mishra et al 1993) and (d) the rise in viscosities are sensitive to the ortho, meta para positions of the groups present in the additive molecules like salicylate (e.g. figure 5 of Rehage and Hoffmann (1991)).

The review remarks 'In terms of these observations, the explanation of the second viscosity maximum is still an unsolved problem'. The main difficulty in rationalizing the characteristics listed above has been due to the non realization of the fact that the molecules like sodium salicylate (SS) are surface active-though to a small extent (Balasubramanian et al 1989). The sodium salicylate molecule is having two -polar groups viz. the COONa and the $\mathrm{OH}$ group both of which have an affinity for water and lie on one half of the molecule. The other half is almost like benzene and therefore there is a clear separation of the polar and the non polar parts. Therefore SS acts like a surface active molecule with a small hydrocarbon portion. This assertion has been verified by a number of experiments (Balasubramanian et al 1989), and has also been shown to have the property of hydrotropy. Once this fact is realized several things follow as a natural consequence. The main point being that CTAB-SS mixed systems should be looked upon as solutions of mixtures of cationic-anionic surfactants, the anionic with a short hydrophobic part. The specificity effects observed with respect to ortho, meta and para positions of hydroxyl group in salicylate systems are simply a consequence of the surface activity of the molecule. The most surface active species would be the SS with $\mathrm{OH}$ group in ortho position next would be the meta and the least surface active would be the para hydroxybenzoate and this is reflected as specificity of the viscoelasticity in these systems (Rehage and Hoffmann 1991). The realization that surface activity of SS like molecules is the key feature in causing the viscoelasticity implies that there would be a whole class of additives which could induce viscoelasticity.

\section{The model}

The main contention of the present paper is to extend and confirm the above observations by demonstrating that by suitable mixing of cationic and anionic surfactants of different chain lengths one can obtain viscoelastic gels or vesicles. The main governing principle being the packing considerations mentioned above. It is known that the $l$ and $v$ parameters for an alkyl surfactant are given by

$$
\begin{aligned}
& l=1.265 \mathrm{n}+1.5 \AA, \\
& v=26.9 \mathrm{n}+27.4 \AA^{9},
\end{aligned}
$$

where $\mathrm{n}$ is the number of carbon atoms in the alkyl chain. Since both $l$ and $v$ are linear in $n$, the ratio $v / l a$ for a given polar head will approach $\sim 2 V a$ for large $n$ 
and therefore there is not much option to vary the parameter $v / a l$ to obtain different structures mentioned above by varying the chain length. On the other hand if one considers a mixture of cationic and anionic surfactants then each cationic-anionic pair would in effect act as a double chain surfactant. If we assume that the cationic surfactant has a longer chain length $l_{0}$ and a volume $v_{0}$ and further, if the combination of cationic- anionic has a polar head area $a$, then the parameter for this combination can be written as

$$
v / l a=\left(v_{0} / l_{0} a\right)\left(1+v_{1} / v_{0}\right)
$$

where $v_{1}$ is the volume of the smaller surfactant-the anionic surfactant-and it is assumed that the length of the surfactant is decided by the longer surfactant. If one assumes that the factor in the first bracket in (3) is approximately $1 / 9$, as is for spherical micelles, then by choosing the volume $v_{1}$ of the anionic surfactant one can vary the ratio in (3) over a fairly wide range to obtain a variety of structures. These arguments will obviously hold good when the longer surfactant is anionic and the cationic is shorter. The work of Kaler et al (1989) on vesicles is an example of this for $v_{1} \simeq v_{0}$. By choosing $v_{1}$ such that $0<v_{1}<0.5 v_{0}$ one can, in principle, obtain cylindrical structures. In fact, such examples, though not realized, exist in literature. Barker et al (1974) obtained viscoelastic solutions by mixing $\mathrm{C}_{\mathbf{s}}$ TAB and SDS. The well studied example of cationic surfactant and sodium salicylate (Hoffmann et al 1985) is another example though the latter is not an alkyl chain.

In the present report we demonstrate the principle of mixing two surfactants of opposite charge to produce various structures by choosing cetyltrimethyl ammonium bromide as the long chain cationic and sodium heptylsulfonate (SHS), sodium octyl sulfate (SOS) and sodium decylsulfonate (SdeS) as the short chain anionic surfactants. It is interesting to observe that (1), (2) and (3) predict that CTAB-SHS and CTAB-SOS should contain cylindrical micelles but CTAB-SdeS should form vesicles. We confirm these effects below.

\section{Results}

Figure 1 shows the zero shear viscosity measured as a function of the concentration of SHS for a fixed concentration of $60 \mathrm{mM}$ of CTAB. These samples apart from being viscous showed viscoelastic effects like recoil of trapped air bubbles. The double peak nature of the figure is characteristic of these cationic-anionic mixture systems (for example, see, Rehage and Hoffmann (1988)) and arises from the fact when one varies the relative ratio one in fact is really varying the charge $Z$ on the aggregates. In view of this variation of $Z$ all the properties, like viscosity, phase diagram etc. which depend on the coulomb repulsions start showing symmetry about $Z=0$ because these effects depend on $Z^{2}$-not on the sign of $Z$. This is the reason why Kaler et al (1989) observed two lobes of vesicular regions and lamellar phases. Similarly the double hump in the viscosity observed by Hoffmann et al (1985) is again the result of this effect. The observation of a two-phase region between the two peaks in the viscosity is also a result of reduced coulomb interaction. Recently Mishra et al (1993) observed two viscoelastic and two nematic liquid phases symmetrically placed about the two phase region in their experiments. Also the results of Barker et al (1974) conform to this rule. Figure 2 shows similar double peaks in viscosity of CTAB-SOS system. These examples clearly show the validity 


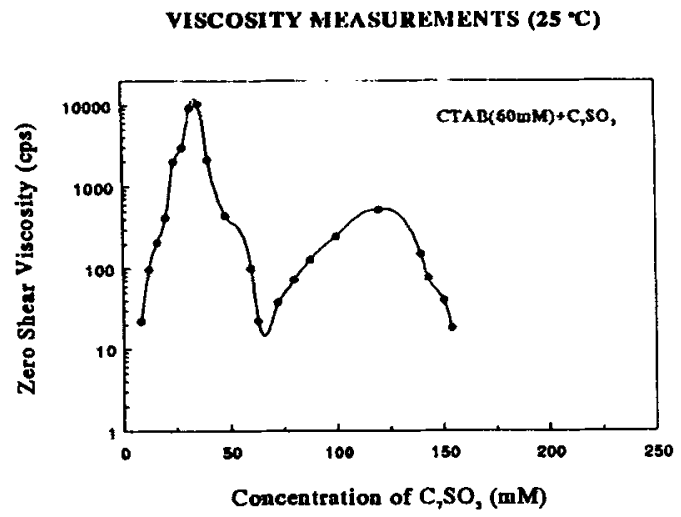

Figure 1. Viscosity extrapolated to zero shear rate of CTAB solution as SHS is added. The CTAB concentration was fixed at $60 \mathrm{mM}$. The double peak in viscosity is similar to the ones reported by Rehage and Hoffmann (1988). These samples also showed strong viscoelasticity and flow birefringence.

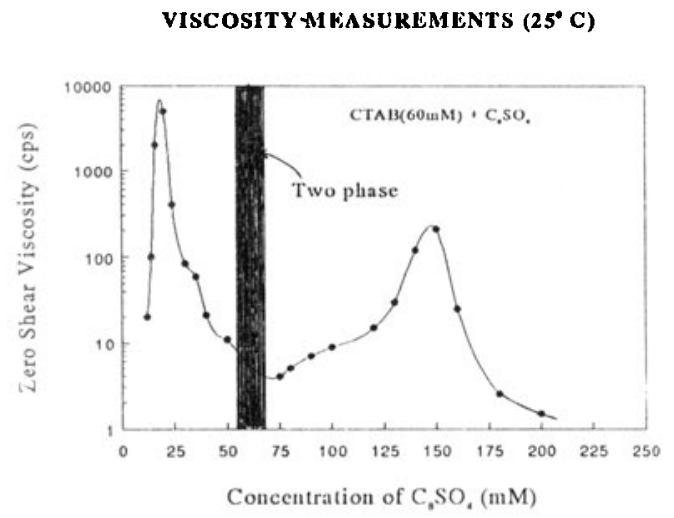

Figure 2. Viscosity of CTAB-SOS system. The presence of two peaks similar to figure 1 and others mentioned in Rehage and Hoffmann (1991) confirms that this is due to surface active nature of additive to CTAB.

of the concepts mentioned above.

The system of CTAB-SDeS formed turbid solutions. The dynamic light scattering results, using Brookhaven Instruments model BI-90, indicated that these solutions consisted of a suspension of particles of diam $150 \mathrm{~nm}$. These results were indicative of the existence of vesicles. Normally one adds Triton X-100 to break vesicles and this worked in our case too. Turbidity of the solutions disappeared on addition of Triton X-100 and the solutions were clear. A fluorescent dye carboxy fluorescene solution was prepared and the two surfactants CTAB and SdeS were added to the solution to produce turbid solutions again. This mixture was dialized to remove the dye outside the possible vesicles. The fluorescent intensity decreased, self absorption 


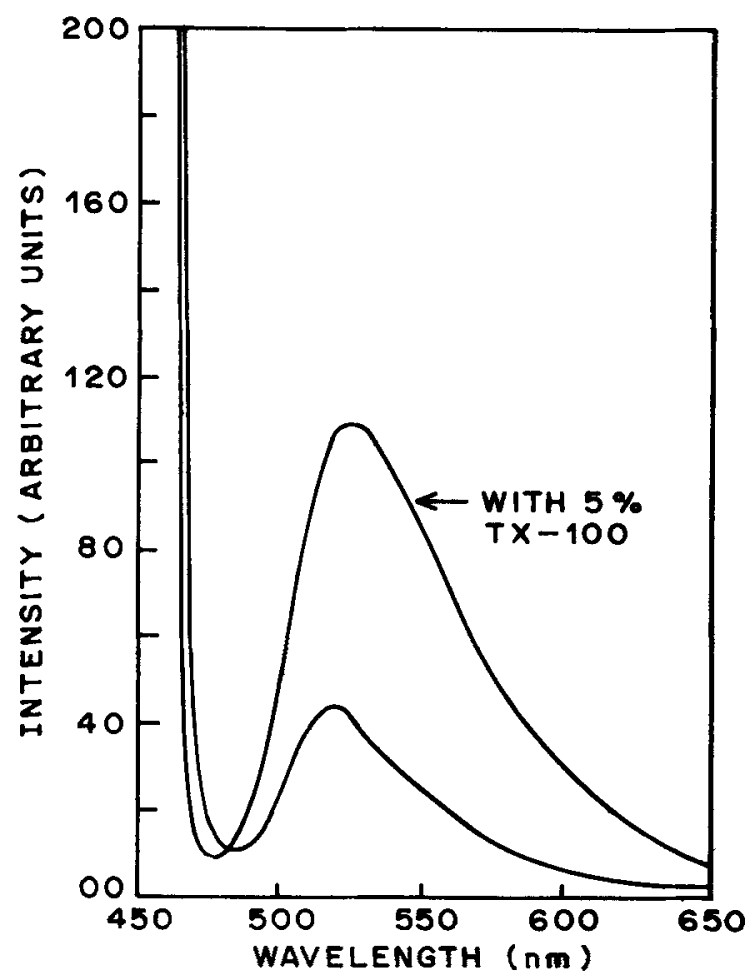

Figure 3. Iluorescence intensity of carboxy fluorescence (Cli). Excitation at $450 \mathrm{~nm}$. The low intensity band is from $C F$ trapped in vesicles. On adding Triton $X-100$ the vesicles break and release the trapped CF and the intensity is enhanced.

being the only mechanism for the dye concentrate trapped inside the vesicles. On breaking the vesicles by addition of Triton X-100 the fluorescence intensity increased indicating the release of the dye. Figure 2 shows the enhancement observed confirming the presence of vesicles. The existence of vesicles is also expected in view of the similarity of the system with that of systems investigated by Kaler et al (1989).

Addition of SdeS to a solution of CTAB with constant concentration of $60 \mathrm{mM}$ also produced two vesicular phases and the transitions

Turbid (vesicles) $\rightarrow$ 2-phase $\rightarrow$ turbid (vesicles),

again indicating the importance of coloumb effects and similarity with the results of Kaler et al (1989).

\section{Conclusions}

These results clearly indicate that the approach initiated by Kaler et al (1989) for vesicles is flexible and can be extended to viscoelastic solutions and possibly to liquid crystalline systems. These results also indicate that with the combination of 
two concepts, viz. the surface activity of the additives like SS, SHS etc. and the coulomb interaction between the aggregates the main characteristics of the viscoelastic solutions mentioned above can be rationalized at least qualitatively.

\section{Acknowledgements}

We are grateful to Drs Ramesh Chander, V M Naik, V V Kumar and Mr R Gopal for discussions and suggestions.

\section{References}

Balasubramanian D, Stinivas V, Gaikar V G and Sharma M M 1989 J. Phys. Chem. 923865

Bangham A D, Standish M M and Watkins I C 1965 J. Mol. Biol. 13238

Barkar C A, Saul D, Tiddy G J T, Wheeler B A and Willis E 1974 J. Chem. Soc. Faraday Trans. I 70 154

Fendler J H 1982 Membrane mimetic chemistry (New York: John Wiley \& Sons)

Hertel G and Hoffmann H 1984 Liquid Cryst. 51883

Hoffmann H, Rehage H, Reizlein K and Thurn K 1985 in Proc. of the ACS symp. on macro and microemulsions (Washington: American Chemical Society) (ed.) D O Shah p. 41

Israelachivili J N, Mitchell D J and Ninham B W $1976 \mathrm{~J}$. Chem. Soc. Faraday Trans. II 721565

Kaler E W, Kanalakara Murthy A, Rodriguez B Z and Zasadzinski J A N 1989 Science 1451371

Mishra B K, Samant S D, Pradhan P, Mishra S B and Manchar C 1993 Langmuir 9894

Mysels K J and Princen L H 1959 J. Phys. Chem. 631696

Nagarajan R 1986 Adv. Colloid. Interface Sci. 26205

Rehage H and Hoffmann H 1988 J. Phys. Chem. 924712

Rehage $\mathrm{H}$ and Hoffmann H 1991 Mol. Phys. 74933

Tanford C 1972 J. Phys. Chem. 763060 